\begin{tabular}{lllllllll}
$\mathbf{R}$ & $\mathbf{O}$ & $\mathbf{Z}$ & $\mathbf{P}$ & $\mathbf{R}$ & $\mathbf{A}$ & $\mathbf{W}$ & $\mathbf{Y}$ \\
\hline
\end{tabular}

ROCZNIKI HUMANISTYCZNE

Tom LXVIII, zeszyt 5 - 2020

DOI: http://dx.doi.org/10.18290/rh20685-1

IRMELA VON DER LÜHE

\title{
GEDENKEN IST VERGESSEN: PARADIGMEN, PROBLEME UND PARADOXIEN LITERARISCHER ERINNERUNG AN DIE SHOAH
}

\begin{abstract}
A b s trakt. Der vorliegende Beitrag präsentiert die literatur-und erinnerungsgeschichtlichen Entwicklungen, die seit dem Ende des Zweiten Weltkrieges für die literarische und literarhistorische Auseinandersetzung mit der Problematik der Shoah bestimmend wurden und an denen sich eine junge Generation von Autorinnen und Autoren in Polen und Deutschland inzwischen kritisch abarbeitet. Der Beitrag geht zuerst den dominierenden Paradigmen des literarischen Shoah-Diskurses, d.h. dem Darstellungsverbot und dem Topos der Unsagbarkeit nach, um dann die methodischen Probleme, die sich aus dem Gebot des Schweigens und dem individuellen und moralischen Zeugnisbedürfnis ergeben, zu erörtern. Die Paradoxien in der literarischen Erinnerungsgeschichte werden im abschließenden Teil an der essayistischen Erzählung von Doron Rabinovici Gedenken ist vergessen (2008) exemplifiziert.
\end{abstract}

Schlüsselwörter: literarische Erinnerung an die Shoah; Paradigmen; Probleme; Paradoxien.

\section{EINLEITENDE GEDANKEN}

In dem Projekt Das Gedächtnis an die Shoah in der polnischen und deutschsprachigen Literatur von Autorinnen und Autoren der zweiten und dritten Post-Shoah-Generation haben wir nach Kontinuitäten und Brüchen, nach Transfigurationen und Subversionen thematischer und formaler, narrativer und poetologischer Prinzipien gefragt, die für eine Literatur des kulturellen Gedächtnisses im allgemeinen und speziell für eine literarische Erin-

Prof. Dr. habil. IRMELA VON DER LÜHE - Freie Universität Berlin, Fachbereich Philosophie und Geisteswissenschaften, Institut für Deutsche und Niederländische Philologie; Korrespondenzadresse: Habelschwerdter Allee 45, 14195 Berlin; E-Mail: vdluehe@zedat.fu-berlin.de. ORCID: https://orcid.org/0000-0003-0036-2688. 
nerungskultur an den Holocaust seit 1945 entstanden sind und die - so die Ausgangsthese - in der polnischen und deutschsprachigen Gegenwartsliteratur der zweiten und dritten Generation einer faszinierenden oder auch irritierenden, einer provozierenden und produktiven Revision unterzogen wurden und werden.

Im Folgenden möchte ich daher die literatur-und erinnerungsgeschichtlichen Entwicklungen, die seit Ende des Zweiten Weltkrieges für unser Thema bestimmend wurden und an denen sich eine junge Generation von Autorinnen und Autoren in unseren beiden, aber auch in anderen Ländern inzwischen kritisch abarbeiten, in gebotener Kürze, aber doch systematisch zu skizzieren versuchen; und ich tue dies - wie im Titel angekündigt - mit Hilfe dreier begrifflicher Leitmotive: Ich frage nach dominanten Paradigmen, nach methodischen Problemen und dabei entstandenen Paradoxien in der literarischen Erinnerungsgeschichte.

Von letzteren, den Paradoxien, kann das Zitat im Titel meines Vortrags bereits eine Vorstellung vermitteln. Es stammt aus einer kurzen essayistischen Erzählung von Doron Rabinovici, auf die ich am Ende zurückkommen werde.

\section{PARADIGMEN: DARSTELLUNGSVERBOT UND TOPOS DER UNSAGBARKEIT}

Eine der folgenreichsten scheinbar normativen Aussagen über Literatur und Kunst nach Auschwitz stammt bekanntlich von Theodor W. Adorno. Sein Diktum aus dem Jahre 1951 „Nach Auschwitz ein Gedicht zu schreiben, ist barbarisch" (Adorno 240) hat in Öffentlichkeit und Forschung, bei zeitgenössischen Autorinnen und Autoren (darunter Paul Celan und Hilde Domin) eine enorme, überwiegend kritische Resonanz ausgelöst. Dabei war und blieb völlig offen, ob Adorno Gedichte „über“ oder „nach“ Auschwitz meinte; ob er für ein Darstellungsverbot plädiert hatte oder ob er lediglich hatte warnen wollen: Vor der Ästhetisierung von Auschwitz, vor dem Konsum des Grauens, vor einer „Verwendung“ des Judenmords für wohlfeile Schauergeschichten; kurzum, ob der Kritiker der westlichen Kulturindustrie, als der Adorno spätestens seit der Dialektik der Aufklärung bekannt geworden war, der Kommerzialisierung von Auschwitz entgegentreten wollte, ohne je das Recht der „Gemarterten“ auf Ausdruck ihres Schmerzes zu bestreiten. Er hat sich bekanntlich später selbst in eben diesem Sinne geäußert und ist daher in meiner Sicht ein visionärer Kritiker jenes sog. „Shoah-Busi- 
ness“, das heute freilich aus ganz anderer politischer Richtung gern attackiert wird.

Aber zurück zu den „Paradigmen“. Wie missverständlich und kontrovers Adornos Diktum auch immer gewesen sein mag, das Paradigma der Undarstellbarkeit, der ästhetischen und ethischen, künstlerischen und moralischen Unsagbarkeit der Verbrechen lässt sich auf Adorno zurückführen. Und natürlich hat es in dieser Bedeutung auch programmatische Bestätigung erfahren, etwa in Eli Wiesels Aussage: Ein Roman über Treblinka ist entweder kein Roman oder er ist nicht über Treblinka. („A novel about Treblinka is either not a novel or not about Treblinka“. Wiesel 7) Dem wiederum liegt die Überzeugung zugrunde, die nicht wenige Überlebende selbst tief geprägt hat, dass der Realität der Lagererfahrung mit den Mitteln der Literatur nicht nur nicht beizukommen, sondern dass es nachgerade ein Hohn sei, dieser singulären Erfahrung literarisch begegnen oder sie auch nur sprachlich „bewältigen" zu wollen.

Von dem Historiker Yosef Hayim Yerushalmi stammt die folgende Einschätzung:

Der Holocaust hat bereits mehr historische Forschungstätigkeit ausgelöst als jedes andere Ereignis der jüdischen Geschichte, doch für mich steht außer Zweifel, daß sein Bild nicht am Amboß des Historikers, sondern im Schmelztiegel des Romanciers geformt wird. (104)

Das Paradigma der Undarstellbarkeit hat mithin zwei sich ergänzende Seiten: zum einen den Gedanken, dass die Verbrechen selbst jede Vorstellung und also auch jede Darstellung übersteigen; zum andern die Skepsis gegenüber den Möglichkeiten der Sprache, und zwar sowohl der alltäglichen als auch der literarischen Sprache, die zur Repräsentation des Erfahrenen und Erlebten schlichtweg ungeeignet sei.

Dem - wie man sieht - ins Aporetische tendierenden Paradigma der Undarstellbarkeit ist bereits sehr früh das genau gegenteilige Paradigma entgegen gesetzt worden; nämlich die wiederum fast normative Überzeugung, die Kunst, der große Roman, das Gedicht könne zum singulären Ort für das Gedenken an ein singuläres Verbrechens werden. Raymond Federman hat dieser Überzeugung Ausdruck verliehen, als er davon sprach, der Holocaust sei das epische Großereignis des 20. Jahrhunderts. (Federman 28) Im wissenschaftlichen Kontext hat das Undarstellbarkeits-Paradigma eine ganz eigene, nachgerade existenziell-poetologische Antwort gefunden; und zwar in der keineswegs rein rhetorisch gemeinten Frage Klaus Laermanns aus dem Jahre 
1998: „Wo denn wären die Millionen Toten zu begraben, wenn nicht in Gedichten?" (Laermann 14)

Einem solchen gleichsam pathetischen Vertrauen in die Validität und Dignität des Gedichts steht ein tiefsitzendes Misstrauen gegenüber, das sich einerseits auf die Singularität der Verbrechen und andererseits auf die prinzipielle Unzulänglichkeit der poetischen Sprache beruft; und beide gehen auch dies sollte deutlich geworden sein - mit einer grundsätzlichen kulturund konsumkritischen Skepsis einher, die in allen Versuchen der Repräsentation das Risiko oder auch die Realität einer voyeuristischen, banalisierenden Zurichtung der Shoah für kommerzielle und/oder gutgemeinte geschichtsdidaktische Zwecke vermutet.

\section{PROBLEME: SCHWEIGEZWANG UND ZEUGNISGEBOT}

Beim Blick auf die literarischen Texte, mit denen wir uns in unserem Projekt näher auseinandergesetzt haben und die heute und morgen Gegenstand einzelner Vorträge sein werden, mag der Eindruck entstehen, die auf Adorno zurückgehende Skepsis gegenüber einer „Literatur nach und über Auschwitz" sei inzwischen einer selbstverständlichen Arbeit am literarischen Gedächtnis gewichen, die aus der Shoah wie fragmentarisch und/oder experimentell, intertextuell und multimedial, faktenorientiert und autofiktional auch immer ein Objekt ästhetischer und/oder theoretischer Moden gemacht habe. Moderne Trauma-Theorien und Konzepte der Post-Memory haben hier allerlei ausgelöst, und damit die Probleme eher vergrößert als gelöst. Ich versuche auch hier den historisch-systematischen Ausgangspunkt zu markieren. In diesem Falle liegt er nicht bei Adorno. Vielmehr betrifft er die bereits in den Lagern und von den Überlebenden empfundene existenzielle und moralische, individuelle und politische Pflicht zum Zeugnis. Zugespitzt gesagt, steht der theoretisch entwickelten Skepsis gegenüber der Erzählbarkeit/Darstellbarkeit der Shoah ein ethisches Zeugnisgebot gegenüber, das für viele Überlebende zum Modus des Überlebens geworden war; wenn überhaupt so galt die Skepsis der Überlebenden weniger der Kraft und Macht ihres Zeugnisses als der Zuhörbereitschaft ihrer Umwelt; und diese Skepsis wiederum basierte auf einer Ahnung, dass die Überlebenserzählungen in ihrer monströsen Dimension den existenziellen und moralischen Horizont derer, für die sie bestimmt waren, schlichtweg übersteigen würden. An der Anstrengung für und am Vertrauen auf die Wirkung ihres Zeugnisses hat das 
die Opfer und Überlebenden freilich nicht gehindert. Im Gegenteil: kaum ein Paradigma wurde so wirkungsmächtig in der Erinnerungsgeschichte an die Shoah wie das Zeugnis, und zugleich wurden die theoretischen und ethischen Probleme rund um das Zeugnisgebot in einer Weise ausdifferenziert, dass sich Dissertationen und Buchreihen dazu publizieren ließen. Freilich liegt hier nicht das entscheidende Problem. Ein Blick auf die Anfänge mag darüber belehren:

„Es ist unser einziger, armseliger Widerstand, dass nichts vertuscht wird, dass alle Zeugnisse erhalten bleiben" - so lässt Ferdinand Bruckner eine Figur seines Theaterstücks Die Rassen schon 1933 ausrufen. (Bruckner 307) Vom Wunsch zu bezeugen, die Erlebnisse von Verfolgung, Tortur und drohender Vernichtung aufzuzeichnen, weiterzugeben und das eigene Leben und Überleben aus diesem Status der Zeugenschaft heraus überhaupt erträglich $\mathrm{zu}$ machen und $\mathrm{zu}$ legitimieren, von diesem Wunsch sind schon zahlreiche frühe Texte des deutschsprachigen Exils bestimmt; ebenso die ersten Lagerberichte aus Sachsenhausen und Dachau, die autobiographischen Aufzeichnungen aus Buchenwald, Theresienstadt und Auschwitz. ${ }^{1}$

Sprechende und für unseren Problemaufriss aussagekräftige Belege finden sich nun freilich vor allem im polnischen Bereich; ich beschränke mich auf wenige Beispiele, die Sie zum großen Teil kennen werden, an die für unseren Zusammenhang gleichwohl zu erinnern ist. Es sind dokumentarische, publizistische und genuin literarische Beispiele, die dem Thema unseres Projekts eine zwar unvollständige, aber doch auch kanonische Vorgeschichte liefern.

\section{BEISPIEL 1}

„Möge mindestens einer übrigbleiben, um der Welt berichten zu können“ (Freiberg 633) - dieser ebenso schlichte wie erschütternde Satz stammt aus

\footnotetext{
${ }^{1}$ Vgl. zum Beispiel Wolfgang Langhoff Die Moorsoldaten (1935), Gerhard Seger Bericht über Oranienburg (1934) und Max Abraham Juda Verrecke. Ein Rabbiner im KZ (1934). Diese drei Texte, aus deren erstem das bekannte und gedächtnispolitisch malträtierte „Lied der Moorsoldaten“ stammt, gehörten im Übrigen zu einem umfangreichen Konvolut von Materialien, das 1936 im Pariser Exil unter anderem von Hannah Arendt zusammengestellt worden war. Zusammen mit der "Ligue Internationale contre l'Antisémitisme" organisierte sie die Verteidigung David Frankfurters; im Prozess gegen den Gustloff-Attentäter sollten dessen Anwälte über Material, also u.a. über die genannten Augenzeugenberichte verfügen können. D.h. mit den Zeugnissen aus den Lagern wurde im Dezember 1936 der - freilich vergebliche Versuch unternommen, den Prozess gegen David Frankfurter zu einem Tribunal gegen den nationalsozialistischen Antisemitismus zu machen. Beweis und Zeugnis - kategorial eigentlich streng zu trennen - liegen hier dicht beieinander.
} 
dem Munde des 17jährigen Berek Freiberg. Mit 14 Jahren war er in das Vernichtungslager Sobibór deportiert worden, musste als „Arbeitsjude“ das Mordgeschehen direkt unterstützen, also den Opfern die Haare scheren, die Leichen wegschaffen oder den SS-Offizieren zu Diensten sein. „Ich glaubte selbst nicht, dass es möglich ist, das zu erleben, was ich erlebt habe" (Freiberg 633-634) - erklärte er 1945. Berek Freiberg beteiligte sich am Aufstand in Sobibór, unter abenteuerlichen Umständen gelang ihm die Flucht. Er war einer von 47 Überlebenden des Vernichtungslagers. Die schon 1944 gegründete Zentrale Jüdische Historische Kommission in Polen bat ihn 1945 um einen Bericht; in jiddischer Sprache entstand er im Gespräch mit Bluma Wasser; es ist eine der ersten und detailliertesten Schilderungen über Sobibór, die wir kennen.

Das in der Forschung sehr kontrovers diskutierte Geschehen in Sobibór soll hier gar nicht referiert werden. Es geht mir lediglich um jene beiden Sätze, die ein seinerzeit 17jähriger Junge zu Protokoll gab und in denen sich die „Probleme“, von denen ich spreche, sprachlich und menschlich in zwei ebenso schlichten wie einprägsamen Überlegungen verdichten: Es möge jemand überleben, um der Welt zu berichten und - zum anderen - mir selbst erscheint unmöglich, dass man erleben konnte, was ich erlebt habe. Wie aus einer solchen Aporie herauszukommen sei, lasse ich dahingestellt; Berek bzw. Dov Freiberg selbst emigrierte nach Israel, arbeitete als Ingenieur und wurde zu einem wichtigen Zeugen sowohl im Eichmann-Prozess des Jahres 1961 als auch im Prozess gegen John Demjanuk.

\section{BeISPIEL 2}

Die existenziell und ästhetisch radikalisierte Variante des Problems der Zeugenschaft findet sich bei einem Autor, den ich Ihnen in Lublin nicht eigens vorstellen muss. Lediglich die für unseren Zusammenhang einschlägigen Äußerungen von Tadeusz Borowski möchte ich in Erinnerung rufen. Die erste findet sich in der Erzählung Bei uns in Auschwitz und lautet: „(Denn) es könnte ja sein, dass wir einmal darüber berichten müssen, dass wir einmal den Lebenden Rechenschaft abgeben müssen und dass wir uns zur Verteidigung der Toten erheben müssen" (Borowski 154).

Sie merken, wie der Zeugnisgedanke sich erweitert und zugleich zum Problem wird: er gilt nicht lediglich den Ungeheuerlichkeiten des Erlebten, er betrifft die „Verteidigung der Toten“" vor den Lebenden; kurzum, das Zeugnisgebot wird überlagert vom Zwang zur Rechtfertigung des eigenen 
Überlebens; vom Erlebten zu berichten erscheint als „Privileg“ gegenüber den Toten, Zeugnisgebot und Überlebensschuld interferieren; und im Falle des Schriftstellers Borowski wird beides durch eine fast zur Gewissheit gewordene Sorge nochmals gesteigert. Sie findet sich in dem zweiten Satz:

„Ich wollte aufschreiben, was ich erlebt habe, aber wer auf der Welt wird einem Schreiber glauben, der eine unbekannte Sprache spricht? Das ist, als wollte ich Bäume und Steine überzeugen“ (Borowski 145).

\section{BEISPIEL 3}

Sie spricht keine „unbekannte Sprache“, spricht auch nicht zu „Bäumen und Steinen“: Hanna Krall, mein drittes Beispiel aus der polnischen literarischen Erinnerungskultur an die Shoah, hat als Schriftstellerin und Publizistin bekanntlich einen ganz eigenen Versuch unternommen, verschütteten Geschichten, verschwiegenen Schicksalen und zerstörten Identitäten auf die Spur zu kommen; Geschichten, die der Holocaust verursacht hatte, die einem Geflecht aus Vermeidung und Verdrängung, Verlust und Vergessen nur mit großem Spürsinn, mit Geduld und Nachsicht, Beharrlichkeit und hoher sprachlich-literarischer Präzision zu entlocken waren. Wie in den beiden anderen Beispielen soll mit wenigen Zitaten das systematische, gleichermaßen existenzielle wie ethische, persönliche wie ästhetische Problem illustriert werden.

Aus Anlass des Bandes Existenzbeweise [2. Ausgabe 1996] hat Hanna Krall ihre Intentionen wie folgt umschrieben:

Jemand muß doch darüber berichten, was mit den polnischen Juden geschehen ist. Ihr Schicksal wird weiterexistieren und beschrieben und erzählt werden. Es ist wie mit den Geschichten in der Bibel, sie sind ewig.

Ich schreibe über eine Welt, die zerstört wurde. Und diese Welt der polnischen Juden war sehr reich. Es war eine eigene Kultur. Sie hatten eine eigene Sprache, mit Literatur, mit Musik, mit Tradition, mit Witzen, mit einem eigenen Essen und so weiter. Es war eine eigene Zivilisation. Ich denke nostalgisch über diese Welt. (Krall 1995)

Man würde solche Sätze gründlich missverständen, würde man sie als Versuch der Sakralisierung des Judenmords, würde man das Bekenntnis zur singulären und überhistorischen Bedeutung der Überlebensgeschichten als proto-religiöse Transformation eines Menschheitsverbrechens ins Schicksalhafte verstehen. Es geht in meiner Sicht und im Sinne eines für unser Projekt 
sehr wichtigen Paradigmas hingegen um literarische, erinnerungsgeschichtliche Rekonstruktionen und Transfigurationen; um die behutsam-entschiedene, bewusste und bescheidene Arbeit an einem literarischen Gedächtnis, dessen vollständige Auslöschung das erklärte Ziel des Holocaust gewesen ist. Es geht zugleich darum, mit Mitteln des Interviews und des Porträts, der archivalischen Grabungsarbeit und der dokumentarischen Darstellung einem Sachverhalt sprachlich-literarisch entgegenzusteuern, den der Historiker Dan Diner ungefähr zum gleichen Zeitpunkt, da Hanna Kralls Arbeiten in Deutschland bekannt wurden, also in der zweiten Hälfte der 90er Jahre, sehr präzise zusammengefasst hat: die Massenvernichtung der europäischen Juden habe ,eine Statistik, aber kein Narrativ” (Diner 126). Mit diesem Befund hat Dan Diner die Folgen der bürokratisch-industriellen Planung und Durchführung des Massenmordes für das Bewusstsein der Späteren, der überlebenden Opfer wie der Täter, zu charakterisieren versucht. Die Gleichförmigkeit, mit der millionenfach Lebensgeschichten in ein tödliches Schicksal ,gestanzt” wurden, habe im Bewusstsein der Späteren eine Leerstelle hinterlassen: „gestaute Zeit“ (Diner 127). Eben diese Leerstelle verweise auf eine „zerstörte Erzählstruktur”, auf die Unfähigkeit des Bewusstseins, die Ereignisse adäquat darzustellen bzw. zu beschreiben. Die theoretischen Implikationen dieser für einen Historiker weitreichenden Positionen sollen hier nicht erörtert werden; welches Ereignis unter welchen Bedingungen je adäquat beschrieben wäre, das ist bekanntlich ein sehr weites Feld. Aber im Lichte von Dan Diners Überlegungen gewinnen die Arbeiten von Hanna Krall nochmals eine besondere Brisanz. Ihre „Interviews gegen das Verschweigen” sind nämlich auch Zeugnisversuche gegen die bloße Statistik, sie sind „Bruchstücke von Biographien”, gesammelt in einem Land, das Hanna Krall den „Friedhof des europäischen Judentums" genannt hat. Bescheiden, aber entschieden hat sie in einem Interview 1995 ihre Arbeit und ihr Selbstverständnis so formuliert:

Ich schreibe immer über den einzelnen. Wir sollten uns trennen von all den Zahlen mit den vielen Nullen. Wir müssen den Nullen reale Gesichter geben. Das ist die Aufgabe des Schriftstellers. Die Zahlen sind vielleicht gut für Historiker und Politiker. Der Schriftsteller ist der seriöse Chronist des individuellen Schicksals ... (Krall 1995)

Ich habe in diesem Abschnitt über „Probleme“ sehr bewusst Beispiele aus dem polnischen Sprachraum und in meiner Sicht repräsentative Personen und Positionen innerhalb der polnischen literarischen Erinnerungskultur aufgegriffen. Gemeinsam ist ihnen eine Konstellation, die selbstverständlich 
auch im deutschsprachigen Raum begegnet, die mir bei Tadeusz Borowski und Hanna Krall freilich eine besondere sprachliche und existenzielle, künstlerische und politische Radikalität zu haben scheint. Das mag ich als Leserin der Übersetzungen zwar nicht wirklich beurteilen können, will aber ein Argument zur Begründung in den Raum stellen, das für die kommenden Diskussionen hilfreich sein kann. Die im deutschsprachigen Raum sehr früh einsetzende Neigung, in immer neuen Anläufen den Unsagbarkeitstopos zu strapazieren, ihn theoretisch zu verschärfen oder literarisch avantgardistisch $\mathrm{zu}$ unterlaufen, ihn methodisch zur Aporie zu treiben und ästhetisch immer neu zu chiffrieren; diesen raffinierten Eingemeindungs-und zugleich Entsorgungsanstrengungen gegenüber einer Problematik, vor deren verbrecherischer Singularität eigentlich alle noch so ausgefeilten intertextuellen Formexperimente und post-historischen Trauma-Theorien zurückschrecken sollten, hat sich - so mein Eindruck und daher meine These aus Anlass von Borowski und Krall - die polnische literarische Erinnerungskultur wenn nicht verweigert, so doch zumindest nicht ausgesetzt. Ich komme damit zu meinem letzten Abschnitt, also den „Paradoxien“ der literarischen Erinnerungskultur.

\section{PARADOXIEN}

Ich illustriere diesen Aspekt wiederum literarisch und zwar - wie angekündigt - mit einer kleinen essayistischen Erzählung aus dem Jahre 2008. Sie stammt von dem 1961 in Israel geborenen und in Wien lebenden Historiker und Schriftsteller Doron Rabinovici, trägt den Titel Gedenken ist vergessen und scheint schon in dieser paradoxen Formulierung alle bisher erörterten Paradigmen und Probleme einer literarischen Erinnerung an die Shoah ad absurdum zu führen. Die Erzählung ist kreisförmig gebaut und wird über das Refrain-artig wiederholte, titelgebende Diktum strukturiert.

Alles beginnt mit dem fiktiven Schriftsteller Egon Katz aus Wien. 1934 hatte er einen Roman „Esther“ fertiggestellt, aus ihm auch gelegentlich öffentlich gelesen, gedruckt wurde er nicht. Katz floh 1936 nach Amerika. „Es ist eine Geschichte von vielen“, wie der Erzähler in insgesamt sechs Stationen, die das folgende Geschehen umfasst, immer erneut wiederholt. Auf der Basis des Romans schrieb der Komponist Fritz Bermann 1937 ein Libretto, das die Geschichte des Manuskripts und die Geschichte seines geflohenen Verfassers nach den Gesetzen der Zwölfton-Musik verarbeitet. Die geplante Aufführung wurde durch den „Anschluss“ verhindert, der Komponist floh; 
mit ihm ging die Partitur verloren, auch von seinem Verfasser verlor sich jede Spur. Freilich blieb die Oper einem Heldentenor im Gedächtnis; Herbert Band, der seine Stimme als Wehrmachtssoldat im Krieg verloren hat, versuchte nach dem Kriege in Österreich völlig vergeblich an Egon Katz und Fritz Behrmann zu erinnern.

Der ehemalige Heldentenor wird als Phantast abgetan, freilich trifft im Jahre 1972 ein Experimentalfilmer, Heinz Amann, auf Herbert Band; der erzählt ihm von Roman und Oper, also wird ein Filmprojekt entwickelt, das sich am zeitgenössischen cineastischen Avantgardismus zu orientieren versucht. Auch der Filmemacher muss bald aufgeben, er engagiert sich in verschiedenen K-Gruppen im Wien der 70er Jahre, nachdem er zuvor alle Muster des Films an den Sammler Willi Kauss verkauft hatte:

„Es ist eine Geschichte von vielen“ steht auch über der nächsten Station, die im Gedenkjahr 1988 spielt. Da geht die Journalistin Anna Senfer der Geschichte des Romans, der Oper und des Films nach und spürt vor allem den österreichischen Emigranten Egon Katz in Brooklyn auf. Der lebt dort seit über 50 Jahren als Hosenfabrikant, weigert sich Deutsch zu sprechen und hat keine Zeile mehr geschrieben. Die Journalistin macht ein Interview, der alte Mann antwortet krudes Zeug, will von seinem frühen Roman rein gar nichts mehr wissen, erklärt stattdessen, er verkaufe keine Bücher, sondern Hosen. Auf das Gedenkjahr in Österreich angesprochen, antwortet der alte Mann mit dem kryptisch-komischen Bonmot, das die Journalistin als Titel für ihren Bericht wählt „Gedenken ist vergessen“. Damit bekommt die Angelegenheit eine öffentliche Dimension, ein Komitee wird gegründet. In die Wiener Gedenkeuphorie platzt allerdings der Artikel eines deutschen Kulturjournalisten, der nachweist, Egon Katz sei nie ein Schriftsteller, sondern bereits in Wien ein Hosenfabrikant, der Komponist Fritz Bermann niemals ein Musiker, sondern lediglich ein Musikliebhaber gewesen, und auch der Sänger Herbert Band habe schon vor dem Kriege keinerlei stimmliche Begabung gehabt. Das folgende Geschehen hat alle Züge einer Groteske, in den Worten des Textes: „Es ist eine Geschichte von vielen: Erst mit diesem Artikel wuchs die vorher kleine Gemeinde für Egon Katz zu einer empörten Bewegung heran, die sich gegen die Verunglimpfung des Schriftstellers und des unauffindbaren Komponisten wandte" (Rabinovici 494). Bürgermeister und Medien schalten sich ein, das Burgtheater veranstaltet eine Matinee für Egon Katz, der nach Wien eingeladen wird, um das Goldene Verdienstkreuz der Republik entgegenzunehmen. Die Veranstaltung steht unter dem Motto „Gedenken ist Vergessen“, die Laudatio hält der zuständige Minister. Die letzten 
Worte der Erzählung lauten: „Wir sind heute nicht hier zusammengekommen, um den Vertriebenen bloß zu ehren, um Egon Katz als Auslandsösterreicher zu würdigen. Wir sind hier wegen seines Lebenswerkes, seiner Dichtkunst, insbesondere aber wegen seines bedeutenden Romans Ruth. In der ersten Reihe war ein greisenhaftes Kichern, ein Meckern, vernehmbar“ (Rabinovici 495).

Nur diese letzte Episode bekommt eine andere interpretative Sentenz, denn jetzt heißt es „Es ist eine einzigartige Geschichte“ - gemeint ist die diskursive Zurichtung einer auf Fiktionen beruhenden Geschichte, die durch alle Kunstgattungen gereicht und am Ende Objekt eines erinnerungspolitischen Medienspektakels wird. Die Ritualisierung und Medialisierung des Gedenkens und der Erinnerung wird - mit Brecht gesprochen - zur Kenntlichkeit entstellt. Der Einspruch des Protagonisten bleibt ungehört, am Ende steht sein Kichern.

\section{SCHLUSS}

Weiter könnte der Weg und abgründiger könnten die Differenzen zwischen den Problemen, die ich im ersten und zweiten Teil dieses kleinen Versuchs skizziert habe und der literarischen Realsatire, die Doron Rabinovici geschrieben hat, kaum sein. Der Erinnerungsdiskurs hat sich professionalisiert und institutionalisiert, er ist integraler und heftig verteidigter Bestandteil des Kulturbetriebs in Deutschland und Österreich, er ist gelegentlich zum selbstbezüglichen Ritual einer bürgerlich-kulturbeflissenen Öffentlichkeit geworden, die sich ihrerseits jenseits von Medialität und Performativität gar nicht mehr zu entwerfen vermag. Die Selbstbezüglichkeit und Selbstvergessenheit dieser betriebsförmig funktionierenden Gedächtnismaschinerie reicht so weit, dass die Stimme des eigentlich „Betroffenen“, jenes im amerikanischen Exil zum Hosenfabrikanten gewordenen angeblichen Schriftstellers Egon Katz, gar nicht mehr gehört wird; dass die paradoxe Wahrheit seines Litanei-haft wiederholten Diktums gar nicht aufgefasst, dass mithin die Medialisierung des Gedenkens ihre Selbstimmunisierung gegen jedwede Infragestellung oder auch nur kichernde Korrektur einschließt.

Dieser Befund ist in seinem komischen Sarkasmus gewiss kein RundumSchlag gegen erinnerungspolitische Anstrengungen; auch widerlegt er zweifellos nicht die hier referierten und in über 50 Jahren ausdifferenzierten skrupulösen Reflexionen über Überlebensschuld und Zeugnisgebot, über 
Schweigeszenarien und literarische Strategien gegen das Zerreden der Unsagbarkeit, gegen die Geschwätzigkeit des Schweigens und die Unzulänglichkeiten des sprachlichen Ausdrucks. Wohl aber könnte dieser Text und deswegen steht er hier am Ende - als literarisches Zeugnis für eine Entwicklung firmieren, die unser polnisch-deutsches Forschungsprojekt zur Erinnerung an die Shoah wissenschaftlich analysieren wollte. Und nicht nur in diesem Falle sah sich die wissenschaftliche Forschung mit der Erfahrung konfrontiert, dass Formen literarischer Subversion der theoretischen Reflexion vorauseilen; wenn nicht sogar überlegen sind.

\section{ZITIERTE WERKE}

Adorno, Theodor W. "Kulturkritik und Gesellschaft." Soziologische Forschungen in unserer Zeit. Ein Sammelwerk. Leopold Wiese zum 75. Geburtstag, edited by Karl Gustav Specht, Westdeutscher Verlag, 1951, pp. 228-240.

Borowski, Tadeusz. Bei uns in Auschwitz. Translated by Friedrich Griese. 2. Auflage, Schöffling \& Co Verlag, 2008.

Bruckner, Ferdinand. Die Rassen. Das dramatische Werk Bd.1. Aufbau, 1948, pp. 225-338.

Diner, Dan. "Gestaute Zeit. Massenvernichtung und jüdische Erzählstruktur.” Kreisläufe. Nationalsozialismus und Gedächtnis, edited by Dan Diner, Berlin Verlag, 1995, pp. 123-139.

Federman, Raymond. Die Nacht zum 21. Jahrhundert oder aus dem Leben eines alten Mannes. Translated by Gerhard Effertz, Greno, 1988.

Freiberg, Berek. "Sobibor." Nach dem Untergang. Die ersten Zeugnisse der Shoah in Polen 19441947. Berichte der Zentralen Jüdischen Historischen Kommission, edited by Frank Beer, Wolfgang Benz, Barbara Distel, Metropol\& Verlag Dachauer Hefte, 2014, pp. 617-652.

Krall, Hanna. "Die Zahlen in Schicksale verwandeln. Gespräch mit der polnischen Autorin Hanna Krall über die Spurensuche nach der verstörten jüdischen Mutter." Freitag. 14 November 1995.

Laermann, Klaus. “'Nach Auschwitz ein Gedicht zu schreiben, ist barbarisch'. Überlegungen zu einem Darstellungsverbot.” Kunst und Literatur nach Auschwitz, edited by Manuel Köppen, Erich Schmidt, 1993, pp. 11-15.

Rabinovici, Doron. "Gedenken ist vergessen. Der Versuch einer Würdigung." 'Auch in Deutschland waren wir nicht wirklich zu Hause'. Jüdische Remigration nach 1945, edited by Irmela von der Lühe, Axel Schildt, Stefanie Schüler-Springorum, Wallstein, 2008, pp. 491-495.

Wiesel, Elie. "The Holocaust as Literary Inspiration." Dimensions of the Holocaust, edited by Elie Wiesel, Lucy Dawidowicz, Dorothy Rabinovitz, Robert McAfee Brown, Northwestern University, 1999, pp. 5-19.

Yerushalmi, Yosef Hayim. Zachor: Erinnere Dich! Jüdische Geschichte und jüdisches Gedächtnis. Wagenbach, 1996. 


\section{WSPOMINANIE ZOSTAEO ZAPOMNIANE: PARADYGMATY, PROBLEMY I PARADOKSY LITERACKIEJ PAMIĘCI O SZOAH}

\section{Streszczenie}

Niniejszy artykuł przedstawia literackie i historyczno-memorialne tendencje, które od zakończenia II wojny światowej kształtowały sposoby zajmowania się problematyką Zagłady w literaturze i do których nadal odnoszą się krytycznie młode pokolenia pisarzy i pisarek w Niemczech i w Polsce. Artykuł rozważa na wstępie dominujące paradygmaty literackiego dyskursu o Szoah, tj. zakaz przedstawiania oraz topos niewyrażalności, a następnie omawia problemy metodologiczne, które wynikają $\mathrm{z}$ nakazu milczenia oraz indywidualnej i moralnej potrzeby zdania relacji. Paradoksy literackiego kształtowania pamięci ukazane zostają w ostatniej części na podstawie eseistycznego opowiadania Dorona Rabinovici pt. Gedenken ist vergessen [Wspominanie zostało zapomniane].

Słowa kluczowe: literacka pamięć o Szoah; paradygmaty; problemy; paradoksy.

\section{REMINISCENCE HAS BEEN FORGOTTEN: PARADIGMS, PROBLEMS AND PARADOXES OF THE LITERARY MEMORY OF THE SHOAH}

\section{Su m m a r y}

This article depicts those literary and historical-memorial trends which, since the end of the World War II, have shaped the methods of approaching the problem of the Shoah in literature and which young generations of writers from Germany and Poland are critical of, even today. In the Introduction, the article takes into account the main paradigms of the literary discourse about the Shoah, i.e. the ban on any presentations of the topic and the topos of its ineffability; it then addresses the methodological problems that arise from this decree of silence and from the individual and moral need to provide testimony. The paradoxes of shaping literary memory are shown in the final part of the article, based on the tale by Doron Rabinovici entitled Gedenken ist vergessen [Reminiscence has been forgotten].

Key words: literary memory of the Shoah; paradigms; problems; paradoxes. 